\title{
December 2017
}

True (A) or false (B):

Selfies 2015: Peer teaching in medical sciences through video clips - a case study

1. The purpose of the class activity described in this study was to integrate the hard and soft skills, a requirement of most curricula.

The use of low-cost simulation in a resource-constrained teaching environment

2. The literature suggests that the high cost of simulation models and the lack of organisational initiatives are possible reasons for the low usage of simulation in surgical training.

Implementing the Angoff method of standard setting using postgraduate students: Practical and affordable in resource-limited settings

3. The Angoff method of setting cut scores typically requires minimal resources and few well-qualified experts in the test domain.

4. To avoid the bias that emerged in similar studies, the researchers in this study chose not to provide correct answers to the judges before the scoring exercises.

Medical students' perspectives on the anatomy course at the University of Zimbabwe

5. Student-led learning of anatomy has been emphasised as a method to overcome the labour- and resource-intensive pitfalls of the traditional didactic training and practical cadaver dissection.

6. The results of the study showed that lectures had a poor fit with teaching aims related to content base.

Clinical undergraduate medical student training at Kimberley Hospital, Northern Cape, South Africa: 'A test of fire'

7. Expansion of the clinical training programme has no impact on clinicians' health service delivery responsibilities.

8. Specialists who participated in this study considered good teaching to entail the appropriate skills, knowledge and attitudes.

Designing interprofessional modules for undergraduate healthcare learners 9. Traditionally, undergraduate medical education has offered many opportunities for exposure to teamwork between healthcare professionals.

10. The steps involved in the development of interprofessional modules described in this article included: knowledge enquiry (identify problem), synthesis (review knowledge), and product tools (adapt knowledge to local context).
Learning outcomes of occupational therapy and physiotherapy students during their community-based education (CBE) attachment

11. The goals of $\mathrm{CBE}$ ultimately increase health professionals' willingness to work in underserved areas.

12. The unavailability of supervisors during CBE activities has been frequently cited as a challenge hindering effective learning during attachments.

Experiences of South African student midwives in following up on the care of a pregnant woman from pregnancy until 6 weeks after delivery

13. Difficulties in recruitment, poor support and financial constraints were reported to be some of the main challenges associated with the followup experience.

14. Based on the literature, it is unclear whether follow-up experiences result in any new learning.

Self-leadership traits of academics to conform to a changing highereducation environment

15. Self-leadership of academics fundamentally refers to being driven by motivation and self-influence to direct oneself towards achieving optimum performance in a situation.

16. Factors such as relationships with peers and peer support are not considered to have any influence on leadership.

Clinical electives at the University of Michigan from the perspective of Ghanaian medical students: A qualitative study

17. Most international electives are skewed towards students from the lowincome nations to resource-rich countries.

18. A common theme that emerged was 'translation' and included paying more attention to infection control, improving 'system factors', and being more empathetic towards patients.

Factors that enable and constrain the internationalisation and Africanisation of Master of Public Health programmes in South African higher education institutions

19. With the increasing internationalisation and globalisation, endeavours to establish and maintain an African character of higher education in South Africa are irrelevant.

20. According to the respondents in this study, there is no clear understanding of or working definition for concepts and processes such as internationalisation and Africanisation as they apply to their professional contexts.

A maximum of 3 CEUs will be awarded per correctly completed test. 